\title{
Recent progress at the Keck Interferometer: operations and V2 science
}

S. Ragland, P. Wizinowich, R. Akeson, M. Colavita, E. Appleby, et al.

S. Ragland, P. Wizinowich, R. Akeson, M. Colavita, E. Appleby, B. Berkey, A. Booth, A. Cooper, S. Crawford, W. Dahl, C. Felizardo, J. GarciaGathright, J. Herstein, M. Hrynevych, C. Koresko, R. Ligon, D. Medeiros, B. Mennesson, R. Millan-Gabet, D. Morrison, C. Paine, B. Parvin, T. Panteleeva, E. Serabyn, B. Smith, K. Summers, K. Tsubota, C. Tyau, E. Wetherell, J. Woillez, "Recent progress at the Keck Interferometer: operations and V2 science," Proc. SPIE 7013, Optical and Infrared Interferometry, 70130B (28 July 2008); doi: 10.1117/12.788070

Event: SPIE Astronomical Telescopes + Instrumentation, 2008, Marseille, France 


\title{
Recent progress at the Keck Interferometer: operations and $V^{2}$ science
}

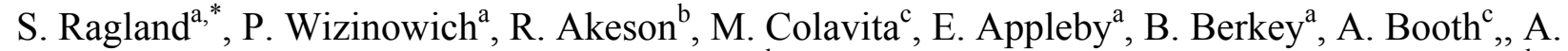 \\ Cooper $^{\mathrm{a}}$, S. Crawford ${ }^{\mathrm{c}}$, W. Dahl ${ }^{\mathrm{a}}$, C. Felizardo ${ }^{\mathrm{b}}$, J. Garcia-Gathright ${ }^{\mathrm{c}}$, J. Gathright $^{\mathrm{a}}$, J. Herstein ${ }^{\mathrm{b}}$,

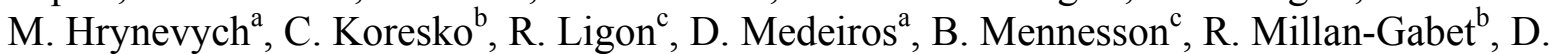

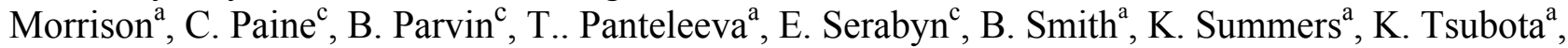 \\ C. Tyau ${ }^{\mathrm{a}}$, E. Wetherell ${ }^{\mathrm{a}}, \mathrm{J}$. Woillez $^{\mathrm{a}}$
}

${ }^{a}$ W. M. Keck Observatory, ${ }^{b}$ California Institute of Technology, ${ }^{\mathrm{c} J e t}$ Propulsion Laboratory

*sragland@keck.hawaii.edu

\begin{abstract}
The Keck Interferometer combines the two 10m diameter Keck telescopes for near-infrared fringe visibility, and midinfrared nulling observations. We report on recent progress with an emphasis on new visibility observing capabilities, operations improvements for visibility and nulling, and on recent visibility science. New visibility observing capabilities include a grism spectrometer for higher spectral resolution. Recent improvements include a new AO output dichroic for increased infrared light throughput, and the installation of new wave-front controllers on both Keck telescopes. We also report on recent visibility results in several areas including (1) young stars and their circumstellar disks, (2) pre-main sequence star masses, and (3) Circumstellar environment of evolved stars. Details on nuller instrument and nuller science results, and the ASTRA phase referencing and astrometry upgrade, are presented in more detail elsewhere in this conference.
\end{abstract}

Keywords: stellar interferometry, visibility measurements, operational efficiency, spectro-interferometry, phase referencing, pre-main sequence binaries

\section{INTRODUCTION}

Keck interferometer (KI) has been jointly developed by the Jet Propulsion Laboratory, the W. M. Keck Observatory, and the Michelson Science Center, and is operational since 2001. The first fringes were obtained in March 2001 and shared risk science started in June 2002. The progress of the KI is presented in the several SPIE papers ${ }^{1,2,3,4,5,6}$ in the past. While the priority of KI during last few years have been the commissioning of the nuller science mode, conducting nuller key science ${ }^{7,8,9,10}$, and performing $\mathrm{V}^{2}$ science observations ${ }^{11,12,13,14,15,16,17}$, several infrastructure and operational improvements have been made during this period in order to improve the observing efficiency and add new observing capabilities in addition to ASTRA developments ${ }^{18}$ which is funded by NSF. In this paper, we discuss the progress at the Keck interferometer in terms of infrastructure and operational improvements, and V2 science results since last SPIE meeting in Orlando, 2006.

The KI is a NASA funded project to perform long baseline interferometry with the two $10 \mathrm{~m}$ diameter Keck telescopes in the near and mid-infrared wavelengths. The physical baseline (B) of KI is $85 \mathrm{~m}$ proving an angular "Resolution" $(\lambda / 2 \mathrm{~B})$ of $\sim 3$ mas at $2.2 \mu \mathrm{m} \& 12$ mas at $10 \mu \mathrm{m}$.

Since the last 2006 SPIE meeting, KI operated on the sky for 57.5 nights - an average of $\sim 29$ nights per year. Of these, 29.5 nights were for V2 science, 3.5 nights (7 half-nights) for V2 engineering, 12 nights for nuller science and 24.5 nights for nuller engineering/shared-risk science. We continued to improve observing efficiency of KI during this 2-year

Optical and Infrared Interferometry, edited by Markus Schöller, William C. Danchi, Françoise Delplancke Proc. of SPIE Vol. 7013, 70130B, (2008) · 0277-786X/08/\$18 $\cdot$ doi: 10.1117/12.788070 
period through operation automation, preventive maintenance of the interferometer sub-systems, staff training and appropriate policy changes. KI is currently used for a variety of stellar astrophysical studies. There are 8 refereed publications during this 2-year period, including 7 papers on V2 observations and 1 paper on nuller observations.

In this paper, we describe new operational capabilities developed during this period which include the extension of $\mathrm{V}^{2}$ science mode in Section 2, recent operational improvements in Section 3, the highlights of current $\mathrm{V}^{2}$ science results in Section 4 and a brief description of the plan for the near future developments in Section 5.

\section{NEW CAPABILITIES}

New medium and high spectral resolution $\mathrm{V}^{2}$ modes in the K-band and a low-resolution $\mathrm{V}^{2}$ mode in the L-band have been implemented at the KI. For this purpose, two new cameras have been added to the basement - a second H \& K-band fringe tracker camera based on a HAWAII detector, and an L-band PICNIC based fringe tracker camera. Each of these cameras is equipped with dedicated beam combiner and alignment stimulus. These improved spectral capabilities are briefed in Section 2.1 \& 2.2. In addition, several sensitivity improvements are briefly discussed in Section 2.3 \& 2.4.

\subsection{Medium/High spectral dispersion mode:}

- K-band medium dispersion mode $(\mathbf{R} \sim \mathbf{2 3 0})$ : This medium resolution (42 spectral channels) $\mathrm{V}^{2}$ mode saw first light in May 2006. This mode has been optimized since then and became available for science in Aug 2006. We present the highlights of the science obtained using this mode in Section 4.

- K-band high dispersion mode ( $R \sim$ 1800): A second fringe tracker camera (Figure 1) has been added as a spare for the $\mathrm{V}^{2} \&$ Nuller modes and as the long integration science camera for the ASTRA project (ASTRA requires two physical cameras - one for fringe tracking at fast rate of $\sim 250 \mathrm{~Hz}$ and the other for science at slow rate of $\sim 2 \mathrm{~Hz})$. The ASTRA project funded a high dispersion grism with 330 spectral channels $(\mathrm{R} \sim 1800)$ for this second fringe tracker which saw first light in April 2008. This mode will be available to the community starting in semester 09A.

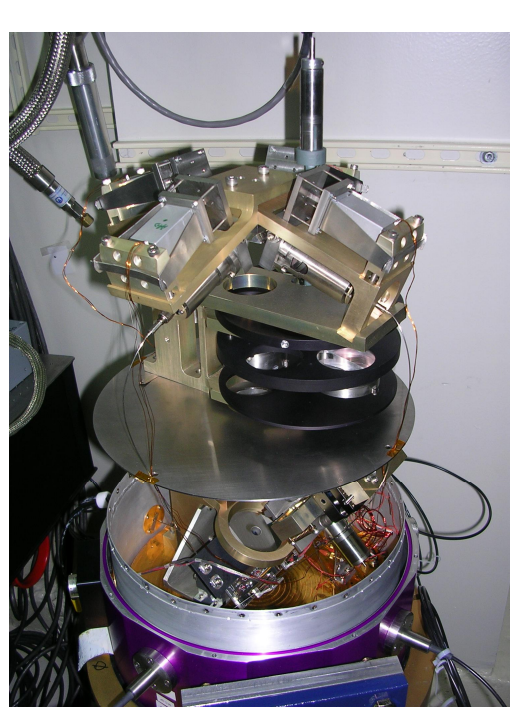

Figure 1 Secondary FT camera

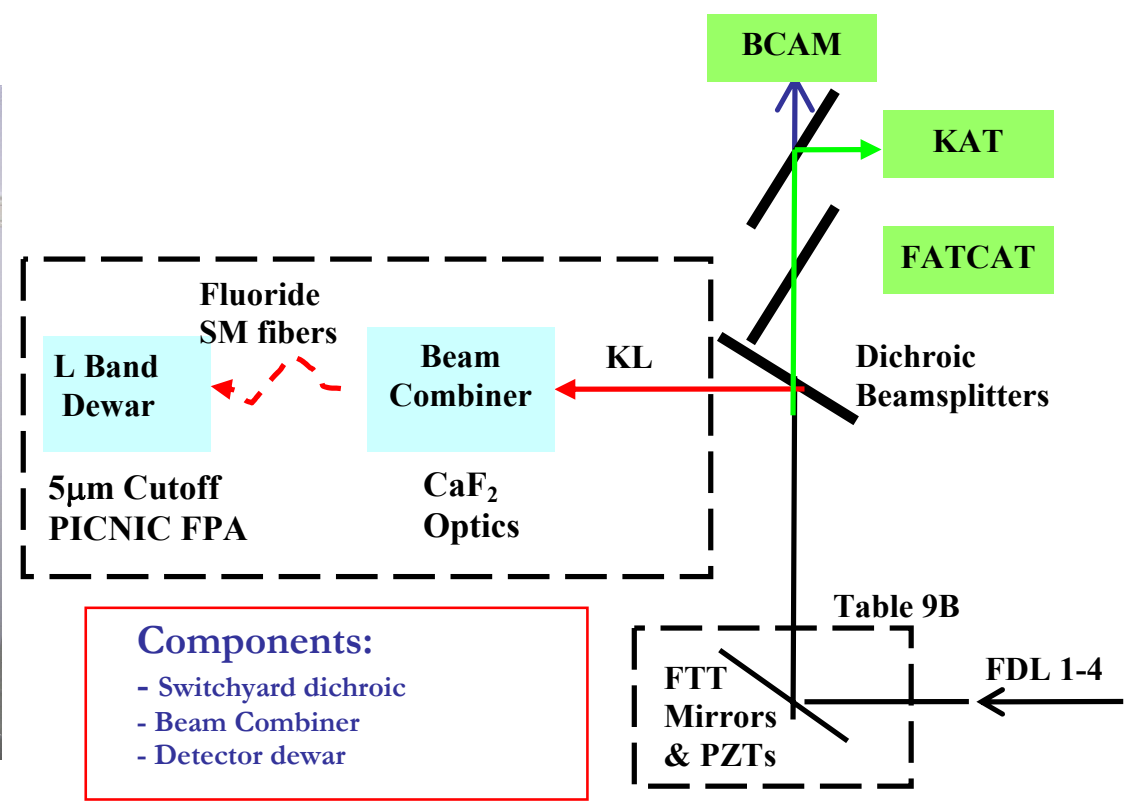

Figure 2 Layout of L-band science instrument 


\subsection{L-band science mode}

The L-band camera, originally developed for the now defunct differential phase mode, is now integrated as a science camera and fringe tracker for the L-band $\mathrm{V}^{2}$ mode (Figure 2). The first fringes were obtained in April 2008 and the mode will become available for shared-risk science in August 2008. Its limiting magnitude is $\mathrm{L} \sim 6$.

\subsection{KI Sensitivity improvements}

- Improved sensitivity of $\mathbf{H}$ \& $\mathbf{K}$-band Science camera: We optimized our fringe-tracking clocks further to reduce detector noise and also increased the integration time to $100 \mathrm{~ms}$. This resulted in about one magnitude improvement in the limiting magnitude of the low resolution $\mathrm{K}$-band $\mathrm{V}^{2}$ mode to $\mathrm{K} \sim 10.3$ magnitudes and $\mathrm{H}$ band $\mathrm{V}^{2}$ mode to $\mathrm{H} \sim 9$ magnitudes (a gain of $\sim 0.5$ magnitudes).

- New angle tracking algorithm: We have implemented a Gaussian algorithm in addition to the standard quadcell centroid algorithm. The Gaussian algorithm improves the centroid at low light levels during astrophysical observations. The light thresholds were reduced accordingly. The current sensitivity is $\mathbf{J} \sim 11.5$ (a gain of $\sim 1$ magnitude).

- Improved H-band angle tracking: H-band angle tracking capability is improved to $\mathrm{H} 10$ by upgrading switchyard dichroics. The first light using a prototype dichroics was obtained in Mar 2008. We are in the process of acquiring dedicated dichroic beamsplitters for this purpose. This improved angle tracking sensitivity would enable KI observations of highly obscured objects which tend to be relatively brighter in $\mathrm{H}$ compared to $\mathrm{J}$.

- Self-phase Referencing mode: This mode is part of ASTRA project ${ }^{18}$, funded by NSF. The first fringes were obtained in May 2008. This mode would improve the sensitivity of high spectral resolution modes significantly - $\mathrm{K} \sim 8$ for $\mathrm{R} \sim 1800$. This mode is expected to become available for shared-risk science in Aug 2008.

\subsection{AO performance improvements}

The limiting magnitude of the AO system was improved by about 1.5 magnitudes during the last two years because of the following two upgrades.

- New AO/IF dichroic beamsplitters: The recently implemented new dichroic beamsplitters which split the light between the interferometer (operating in the near \& mid-infrared) and the AO wavefront sensors (operating in the visible wavelengths) improved the throughput in the visible by $\sim 50 \%$.

- Wavefront controller upgrade: While not part of KI development, we list them because of the positive impact on interferometric observations. As a part of this upgrade new low noise cameras were installed, improving the sensitivity of the wave front sensors ${ }^{19}$. The KI project funded the AO chopping implementation for this upgraded wavefront controller since this feature is used by KI for the nuller operations.

\section{OPERATIONAL IMPROVEMENTS}

\subsection{Beam-train alignment automation}

3.1.1 Daytime alignment

Beam-train alignment automation is essential for (1) easy operations, (2) manpower reduction, (3) operator-independentperformance, (4) minimized operator errors, (5) maximized throughput and (6) maintaining a stable instrument with an optimized performance. Beam-train alignment task involves carefully threading the beam from the science camera (stimulus for the science camera) to the telescope (stimulus for the AO system) by adjusting 8 optical elements for the $\mathrm{V}^{2}$ mode (16 for the nuller mode) to optimize the pupil location and the beam direction. In total, $\sim 32$ optical elements are adjusted either manually or through various operational tools for $\mathrm{V}^{2}$ observing run preparation and $\sim 98$ optical elements are adjusted for a nuller run, not counting focus adjustments in all our cameras and optics in the AO system. 


\subsubsection{Nighttime alignment}

In order to help ease the large effort required to get the Nuller aligned and configured, many of the night time alignment tasks have been automated within the auto-alignment (AA) framework ${ }^{1}$. The first of these tools to be implemented under AA was the night time long delay line (LDL) move. For day time engineering alignments when there is no careful alignment to maintain, the LDL carts can be moved from a simple GUI which takes a new LDL position in meters and a mode option - either a retro reflection of M11 mirror or a coude alignment to link the basement up to the telescopes. At night, the requirement to return the starlight beam to 0 ".6 on the angle-tracker camera is also imposed. This angular alignment tolerance requirement placed a large realignment overhead of 20 minutes per realignment in the simple twobeam $\mathrm{V}^{2}$ case. However with the addition of the AA automation this time has been reduced to about 5 minutes in V2 mode and about 10 minutes in the nulling mode. The procedure itself has also been simplified enough that multiple operators have now successfully performed these alignments during an observing night.

Two other Nuller specific alignments ${ }^{7}$ have been implemented in AA as well. The flux peak alignment is designed to optimize the pointing of Nuller. This was initially performed by putting a 1-sided chop onto the AO tip/tilt mirrors and manually moving mirrors in the Nuller beam train to peak up the demodulated flux. Then once a maximum flux was found on all of the Nuller ports (when we used this alignment technique 2 ports were in use now all 4 are in use) the chopping was rotated 90 degrees and the flux peak was repeated. Under the new scheme a four side chop is put on the AO tip tilt mirrors such that the mirror moves in the following motion, (right-center-left-center-up-center-down). A virtual centroid is computed from the difference between left-center vs right-center and center-up vs center-down. This system is then used to drive the virtual centroid to the center, allowing for a much quicker more robust alignment technique for the starlight on the Nuller. Alignments went from taking an average of about 23 minutes with some taking nearly 40 minutes, to consistently taking less then 15 with some taking as little as 8 minutes.

The Nuller shear alignment was improved both by the creation of an alignment script and to a greater extent by the addition of shear sensing cameras. The original technique devised for controlling nulling shear was to use a beacon laser from the basement to set a reference mark on a target in the DSM during the day. For each star visited during the night the targets were reinstalled and the optics realigned to return the Nuller beacon laser to the daytime reference mark. The target in the beam had the effect of shuttering out the star light, so the angle tracker control loop must be idled during the shear alignment. The new technique involves placing a pickoff dichroic behind an optical transmissive, IR reflective dichroic in the DSM, and directing some of the laser beacon light onto a camera focused to the same plane as the DSM target. With these shear sensors the starlight beam no longer needs to be shuttered during the night and the angle tracker loop can remain closed during the shear correction. This is important because the behavior of the basement mirrors is dependent on the angle tracker providing corrections for the telescope pointing. Automation of this procedure has also had the expected efficiency gains; previously a shear alignment often took about 6 minutes the alignment is now routinely done in about 2 minutes. The same argument about expert users also applies, it's faster and anyone can do it.

\subsubsection{Near-future upgrades}

With the new L-band and ASTRA modes coming online at Keck there will likely be an opportunity for further improvements to observing efficiency by alignment automation. Currently we plan to re-implement a version of the Nuller pointing for the L-band science mode. Further in the future the ASTRA project will require multiple nighttime reconfigurations to calibrate out instrument errors and provide precision astrometry. We anticipate that automation of this alignment will be beneficial.

\subsection{New operational tools}

We have developed several operational tools during the last two year period. These new tools continued to improve our operational efficiency because of easy usage compared to the previous version of the tool or manual procedures, and their monitoring capabilities that we found extremely helpful through our prior experience in designing and developing other operational tools such as 'aoOper' and 'Ifoper'. We discuss below two major tools. 


\subsubsection{IFSys.py}

The 'IFsys' operators tool (Figure 3) was created as a replacement for the original start2.1 command line script. It provides all of the features available from the cshell script, as well as process monitoring, automated RTC system reboots, dynamic configuration, and multiple simultaneous VxWorks console port access.

The IFsys tool uses a distributed server and client GUIs to allow users to perform the many tasks and operations. Both the server and GUI are written in Python. The server (IFsysServer) maintains the list of applications and where they're running by querying all hosts on startup. Multiple client GUIs can be run simultaneously on any operational summit host with full control and communicate with IFsysServer which they connect to via the CORBA naming service. If the server is not running when the first client GUI is launched, the GUI will automatically start one.

The majority of the commandable operations executed through the GUI widgets by clicking on the labeled buttons are designed to report back a device or application status as a color code. In general there are two states that operators need to be concerned with. Red indicates that the device or application is not running or cannot be accessed (bad for night time observing) and grey indicates that the device or application is running (good for night time observing). Depending on the button, it will connect you to interactive sessions, run scripts, launch other GUIs, allow you to start / re-start applications, etc.

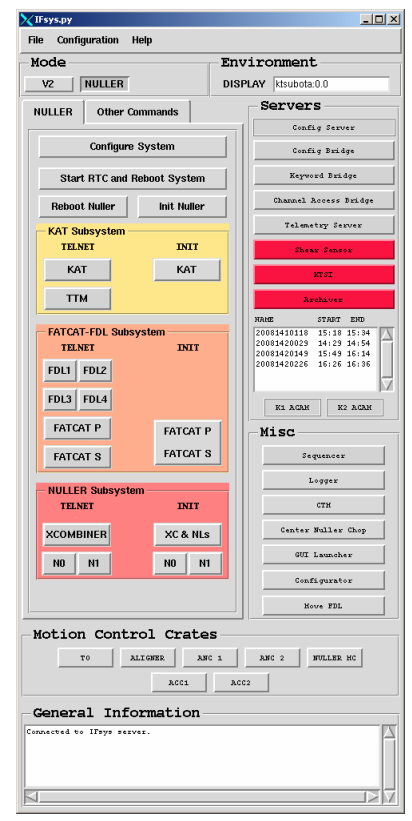

Figure 3 IFsys.py GUI

\subsubsection{Nuller Sequencer}

The nuller sequencer controls the nuller from fringe acquisition, through mode transition, to data collection. The Nuller Sequencer GUI (Figure 4) is layered on top of the Nuller Sequencer application and allows the operator full control over the Nuller Sequencer. The GUI is started after the Nuller Sequencer and calls the sequencer Connect method passing its CORBA IOR. The sequencer Connect method then turns the IOR string into a CORBA object reference to the GUI.

The Nuller Sequencer is a multithreaded finite state machine based on a state machine created using the State Machine Compiler [http://smc.sourceforge.net/] and the Keck developed SMC server and client library. full capability sequencer consist of 3 levels. At the bottom is the SMC generated python state machine code and a derived Sequence class that provides the functionality for each state. Like most sequencers, the nuller sequencer consists of several state machines (Sequencer classes) running in different threads.

The Sequence class is the base class and above the Sequence is the SequenceManager. The SequenceManager creates the threads needed by the application and handles the communication between them. Each thread is named as it is created and the SequenceManager class keeps a dictionary of the threads so it can reference them by name. The SequenceManager also provides the CORBA interface for the application.

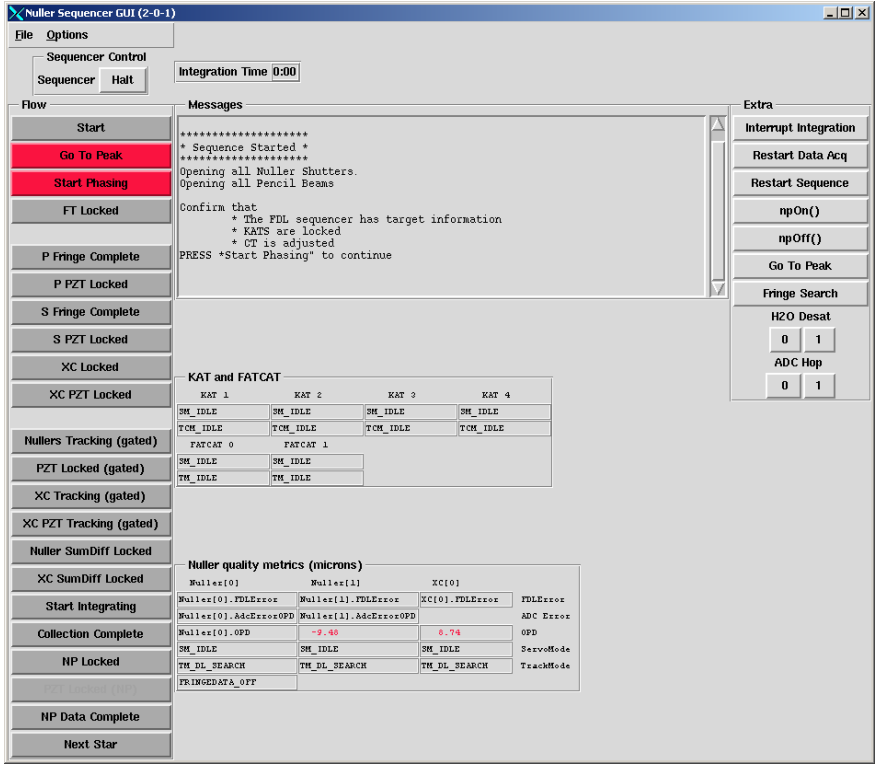

Figure 4 Nuller Sequencer GUI 
The SequenceManager class is a base class for the NullerSequenceManager which manages the sequence threads. Above the manager is the NullerSequencer itself. The NullerSequencer does little more than finding the NameServer, the TelemetryServer, creating the SequenceManager and calling its CreateSequencers method.

While the codes for the threads are identical, the roles they play are not. In the Nuller Sequencer, there is a main thread that once started, kicks off the other threads to do the actual processing. Each sequence thread also publishes its current state as a telemetry item.

\subsection{Analysis tools}

- $\quad$ N0 Tool: N0 provides a method to look back into archived data and produces a set of power spectrum plots covering all of the major interferometer subsystems. The tool is a highly specialized collection of shell scripts, c routines, and a custom Excel spreadsheet. It has slowly evolved to become an over all performance monitoring tool which can provide an at a glance snap shot of performance. It is often used to hunt down new vibrations or to compare performance of the interferometer with a previous epoch.

- Seq2Psd Tool: Seq2Psd is an AA derived tool which extends the functionality provided in the RTC GUIs. Seq2Psd.py can subscribe to any telemetry channel and produces a power spectrum of the data set which is published back to the telemetry server and available on any client GUI. Further since the telemetry handling and the mathematical processing are well separated, Seq2Psd can easily be extended to fit specific real-time analysis task.

- In addition, tools to diagnosis the performance of Fast Delay Lines (FDL) and accelerometer performance through power spectral analysis have also been developed.

\subsection{Procedural improvements}

The observatory has made strides to improve the availability of backup power within the facility. The interferometer team took this opportunity to create a comprehensive procedure for powering up/down all of our equipment and recovering from a power outage. This living document is part of the operational procedures located on a Twiki page.

Maintaining a stock of critical spares is necessary to keep a system of this complexity functioning. A comprehensive list of parts has been compiled and ranked according to criticality, availability, cost and probability of failure. The parts inventory is maintained in a database that contains the information about the part, including storage location, manufacturer and vendor information, drawing numbers, notes on status, etc. To date, we have been able to prevent any significant loss of observing time due to lack of spare parts.

Identifying maintenance tasks and performance tests are another ongoing area of improvement. In order to preserve operational reliability, a number of tests and maintenance tasks are repeated on a periodic basis. In some cases, tests are performed to determine if maintenance is necessary; in other cases, tasks are performed on a routine basis. Testing examples include an overnight test to analyze the Fast Delay Line jitter performance and power spectrum analysis of the accelerometer data. Both of these tests have caught degraded performance that would have impacted observing. Maintenance tasks include inspection/lubrication of motion stages, replacement of optics, vacuum service on cryostats, delay line track inspection/ cleaning and oxygen alarm calibrations.

The continued use of week-before-run and day-of-run checklists has kept the run preparations focused. These checklists also provide historical information on alignment and camera performance, as a number of values (flux \& noise for example) are entered on the checklist. This document continues to be updated as the preparation procedures evolve and obscure failure modes are identified.

The configuration control process at the KI is successful in protecting the operational system while enabling the development of new capabilities. A configuration control board (CCB) meets bi-weekly to review the engineering change requests (ECR) and field change notices (FCN). There were 508 new ECR/FCN, $543 \mathrm{ECR} / \mathrm{FCN}$ closed and 37 
canceled since 1 June 2006 (Software: New: 505, Closed: 458, Canceled: 29; Electronics: New: 46, Closed: 40; Canceled: 1; Mechanical: New: 27, Closed: 31, Canceled: 5; IF operations: New: 20, Closed: 14, Canceled: 2). All modified IF subsystems are captured and frozen in-situ then rebuilt from scratch on a separate host after every run.

\subsection{Cryogenic camera autofill}

The cryogenic cameras used within the interferometer require daily fills with $\mathrm{LN}_{2}$. For a number of reasons, it was determined that an automated system should be implemented. As a result of the physical separation between the supply dewars and the instruments, and the volume of cryogen required, this system has proven to be a challenge to implement. Due to its critical function, a significant amount of effort has been focused on improving the performance of this system. There are currently four cameras with a daily requirement of about 45 liters of liquid nitrogen. The storage dewars are located on the K2 dome floor. To transport the cryogen, vacuum jacketed supply lines $\sim 50-80$ feet long were installed to the basement. The system is controlled by a PLC which also provides dewar status monitoring, safety checks and emergency shutdown functions.

Recently, an improved web-based logging/monitoring tool was developed to monitor the autofill system and trend the performance of the basement cameras. This system provides a timeline of daily events (fills, warnings, etc.) and temperature graphs. The display can be scaled to show a month of data so trends can be easily seen. Hardware changes have been implemented to improve cryogen delivery. The original system design relied on the pressure build system internal to the cryogen storage dewar to provide enough pressure to deliver the liquid to the basement. This proved unreliable. Delivery pressure would vary greatly between dewars and how much cryogen was in the dewar. The system was modified to use a separate dewar as a pressure source. Gas from this dewar is regulated and provides the pressure source for the two liquid supply dewars. Having a constant delivery pressure has significantly reduced the fill times, decreased losses in the piping, and reduced the necessity for user intervention. Monitoring the system has shown that a degrading vacuum in any of the ten supply line segments will also severely impact performance. A quarterly preventative maintenance task has been started to renew the vacuum in the cryogen supply lines. Ion pumps are also installed on three of the four cameras to help maintain their vacuum and extend the service interval.

To eliminate the possibility of electrical interference, custom isolators were designed for the vent side of the dewars. The initial design used off-the-shelf isolators, but these would bridge with ice in a few days, defeating the isolation. This would result in an increase in detector noise. The new isolator design incorporates fins which increase the surface area and heat transfer, keeping the electrical isolation intact.

\subsection{Minor upgrades}

The KI computing environment is upgraded to a more stable configuration. We have in place a redundant system such that we could continue observing in the event of the failure of any one machine. There are software tools in place that allow us to do much of the system role assignments from a GUI, on-the-fly. All mission-critical disks are RAIDed, with e-mail notification when problems are detected, and subject to a tested back-up policy. We moved all Sun servers and disks computers from the basement to the Keck 2 computer room for better access and improved conditions.

We ported over to PostgreSQL [http://www.postgresql.org/] after many years of Sybase [http://www.sybase.com/] use when WMKO decided to move away from Sybase. Since RTC was originally developed for PostgreSQL and was still supported by JPL it was the obvious choice. Also, PostgreSQL is open source and as such has no yearly license or maintenance fees. A big advantage we gained after the port is that our Configurator now caches the database in less than half the time on average than Sybase, $<15 \mathrm{~min}$ vs $>35 \mathrm{~min}$.

The clock generation code was updated to add more parameters to the clock header and the camera gizmo was updated to get all required information out of the .clk file rather than getting through configuration database. As the software has evolved, so have the processing power requirements. Several of the real-time systems received CPU upgrades or an additional CPU to keep up with the demand. Some of the remaining beam optics used for routine alignments were upgraded with electronic actuators. The video servers that provide images of the alignment targets were all upgraded to 
current models. The PZT actuators on the M14 mirrors used for the low-frequency offload of tip/tilt correction were upgraded. The original actuators had excessive stiction. A raster mirror was added to the spectral side of the fringe tracker to improve fiber alignment. The Fast Delay Lines received a dither waveform generator upgrade and a trigger DAC upgrade; these upgrades reduce the CPU overhead and add the flexibility to change the dither parameters on the fly. A robust cable system was designed for the accelerometers on the tertiary mirror. The connectors on the previous system were prone to failure during reconfiguration of the telescope. The automated alignment targets on the Dual Star Module were upgraded to be more repeatable and less failure prone. There continue to be elusive sources of detector noise. Several modifications have been made to eliminate suspected sources of noise. Isolation of the fringe tracker internal PZT signals and the autofill vent isolator (discussed in the autofill section) are examples of this effort.

In order to improve the basement seeing, we air-sealed the Coude (basement-to-telescope) crypt windows and installed crypt doors. We have also installed Coude light pipes on K2 telescope between M5 \& M6 mirrors. We deferred the installation of light pipes on $\mathrm{K} 1$ telescope because of inconclusive results on $\mathrm{K} 2$ implementation. A seeing tool based on closed loop wave-front controller data was implemented by AO team. This provides real-time Strehl monitoring which enables us to optimize the choice of science observing programs for given seeing conditions.

\subsection{Statistics on operational efficiency improvements and challenges}

We have improved operational efficiency significantly during last year. The factors responsible for the improved efficiency include (1) well defined policies for run preparation and run support, (2) careful scheduling of personnel and instrument resource, (3) alignment automation, (4) new Operational tools, (5) cleaning up operational documents/procedures and (6) appropriate staff training. Observing run preparations for the last several runs have take fewer resources than before. Basement alignments can now be performed with a single person on the summit, where two were previously required. Week-before-the-run Coude beam-train alignments are performed only once before each run compared to three times in the past ( $\sim 66 \%$ gain in telescope \& staff resource for this task). Nuller breadboard alignments performed every night of a nuller run now take twenty minutes vs. two hours under the old alignment procedure. We have also gained the sky time by $\sim 23 \%$ for nuller nights and by $\sim 5 \%$ for V2 nights. It is safe to say that the improved nighttime observing efficiency enables fully calibrated nulling observations of 3 science stars per night.

We experienced a few challenges during the last two years at KI. The Big Island of Hawaii experienced an earthquake of 6.8 magnitude on 15 Oct 2006 which damaged the telescopes and misaligned the AO bench. The interferometer itself suffered very little harm - a few optical misalignments - being at the basement of the telescope building. The telescopes were back on the sky (with certain restrictions) for a successful $\mathrm{V}^{2}$ run 19 days later. One of the stabilized metrology laser aged and failed during observing resulted in a loss of sky time. The failure mode was a loss of wavelength stability which made trouble shooting difficult. Subsequently all stabilized lasers were replaced as a precautionary measure.

\section{4. $V^{2}$ SCIENCE RESULTS}

The visibility $\left(\mathrm{V}^{2}\right)$ mode combines the adaptive optics corrected beams from the two Keck telescopes for measuring the fringe contrast in the near-infrared atmospheric windows. We had 21 observing runs since 1 June 2006 - 29.5 science nights ( $16.5 \mathrm{~V}^{2}$ Science \& 12 Nuller Science) and 28 nights for engineering ( 3.5 for $\mathrm{V}^{2}$ ( 7 half-nights), $24.5 \mathrm{Nuller}$. The on-sky observing efficiency for $\mathrm{V}$ nights during this period is $\sim 62 \%$. We lost the rest of the sky time to weather and instrument problems $(\sim 26 \%$ for weather; $\sim 4 \%$ for Telescope/AO problems; $\sim 7 \%$ for Interferometer instrument problems $(\sim 2 \%$ if we exclude one night we had a major hardware failure). There are seven refereed articles published from this instrument since the last 2006 SPIE meeting. These results could be classified into three broad categories namely, (1) protoplanetary disks, (2) pre-main sequence star masses, and (3) circumstellar environment of evolved stars. We briefly discuss the results obtained in these three areas using KI.

\subsection{Protoplanetary disks}

4.1.1 Water vapour and hydrogen in the terrestrial-planet-forming region of a protoplanetary disk 
The dust and gas around the young star MWC 480 were resolved from KI observations (Eisner 2007; Figure 3). Strong gaseous emission was detected inside the dust sublimation radius, offering a possible cause of inward migration of planets to very small orbital radii like those observed for some exo-planets. Spectral signatures of compact water and Hydrogen gas were also detected. The Hydrogen gas appears to trace accretion onto the central star. The large amounts of water in the habitable zone may contribute to the formation of habitable planets.

The fundamental measured quantity, the spectral spatial coherence, shown as squared visibility, is proportional to the inverse of the characteristic size of the emitting region. In this paper the data are used to constrain the dimensions of a simple model. The measurements were made possible by the large collecting apertures and the adaptive optics of the Keck telescopes feeding light to the KI.

\subsubsection{T Tauri Inner-Disks}

The inner edges of the dusty disks around a sample of low-mass (including solar-type) T Tauri stars were resolved using KI observations (Eisner el al. 2007; Figure 4). The interferometer data were combined with new spectroscopic and photometric observations to provide a more complete picture of the inner disk regions. The most interesting result of this survey is that the inner disks around low-mass stars appear to have larger inner holes than expected from simple disk models where truncation is due to dust sublimation. Rather, in these low mass stars, the lower relative mass accretion rates may lead to qualitatively different physics, since the balance between the stellar magnetic field and the force of inwardly accreting material can differ from those of better-studied, higher-mass young stars.

For most young stars observed to date with near-infrared interferometers, the measured sizes of the emitting regions are consistent with predictions of disk truncated at sublimation radius. For many low-mass stars, they are larger than predicted by these models, indicating that disks are truncated by something other than dust sublimation. Magnetospheric truncation suggested, since objects with larger inner disk sizes also have low accretion rates.

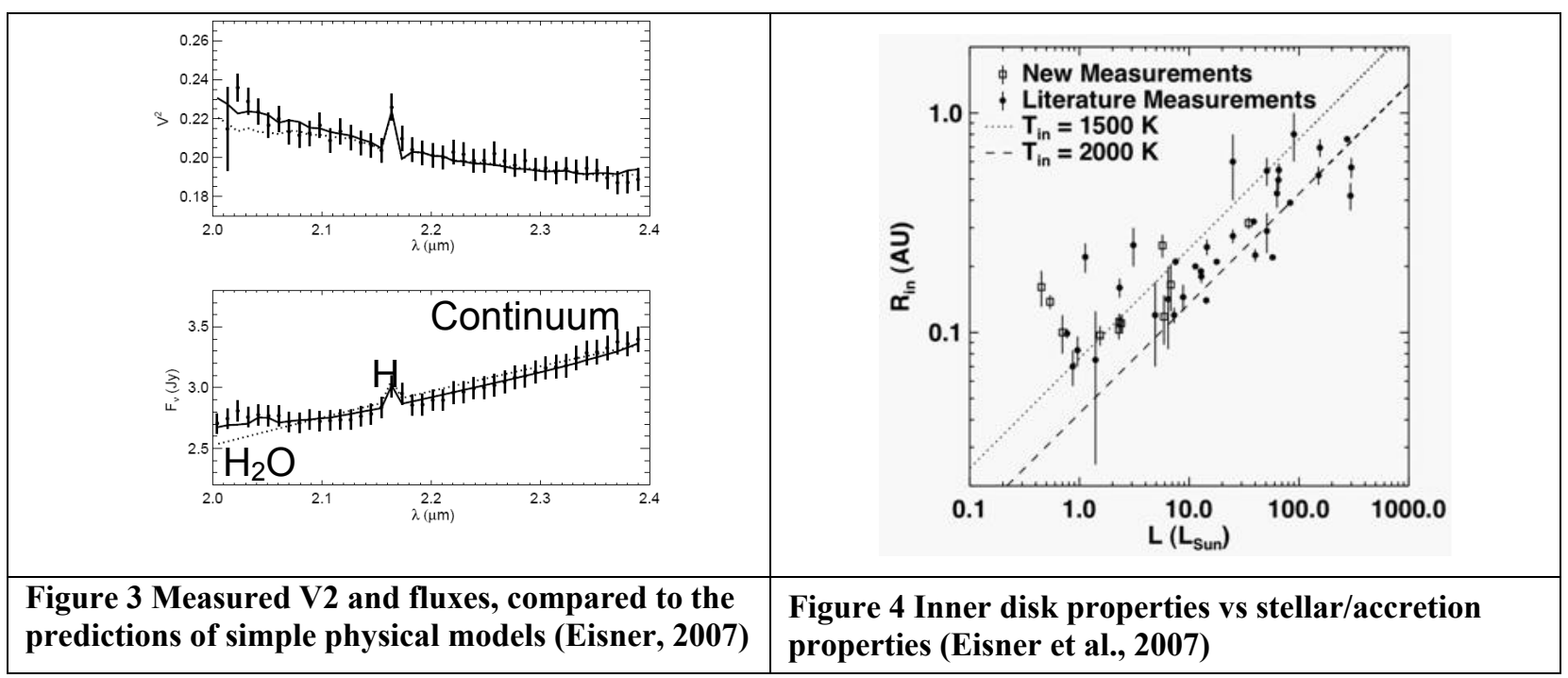

\subsection{Pre-main sequence (PMS) binaries}

The mass of a star determines how a star evolves throughout its lifetime. Accurate masses and ages are needed for understanding a region's star formation history and initial mass function. Masses and ages of PMS stars are commonly determined from a star's location in the HR-diagram. Different sets of evolutionary tracks yield discrepant results, particularly for $\mathrm{M}<1 \mathrm{M}_{\text {sun }}$. High precision dynamical masses are required to calibrate the evolutionary tracks. In combination with RV measurements, astrometric orbits yield physical orbits \& individual masses. The orbital and 
physical parameters of the young binaries V773 Tau A and Haro 1-14c are constrained using KI observations (Figure 5 \& 6) and compared with PMS modes. The $\mathrm{Y}^{2}$ models agree better with the V773 Tau A observations compared to other available models, and the age of V773 Tau A is estimated to be $3 \mathrm{Myr}$ (Boden et al. 2007). Based on comparison with evolutionary tracks, the age of Haro 1-14c is estimated to be 3-4 Myr (G. H. Schaefer et al. 2008). More recently, A. Boden et al. (2008) resolved a warm dust component of the binary system DQ Tau and derived the direction of orbit rotation on the sky.

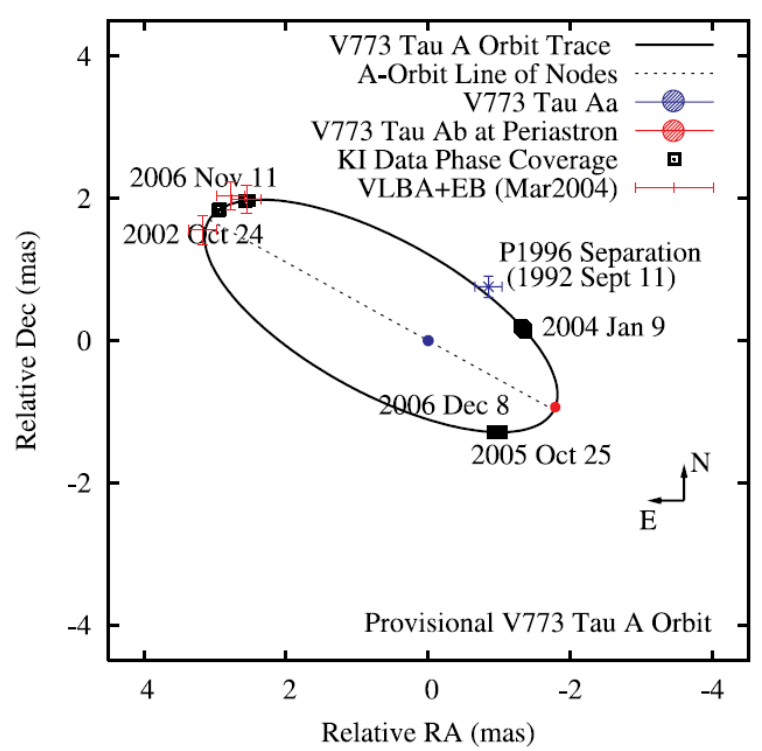

Figure 5 V773 Tau A Orbit (Boden et al., 2007)

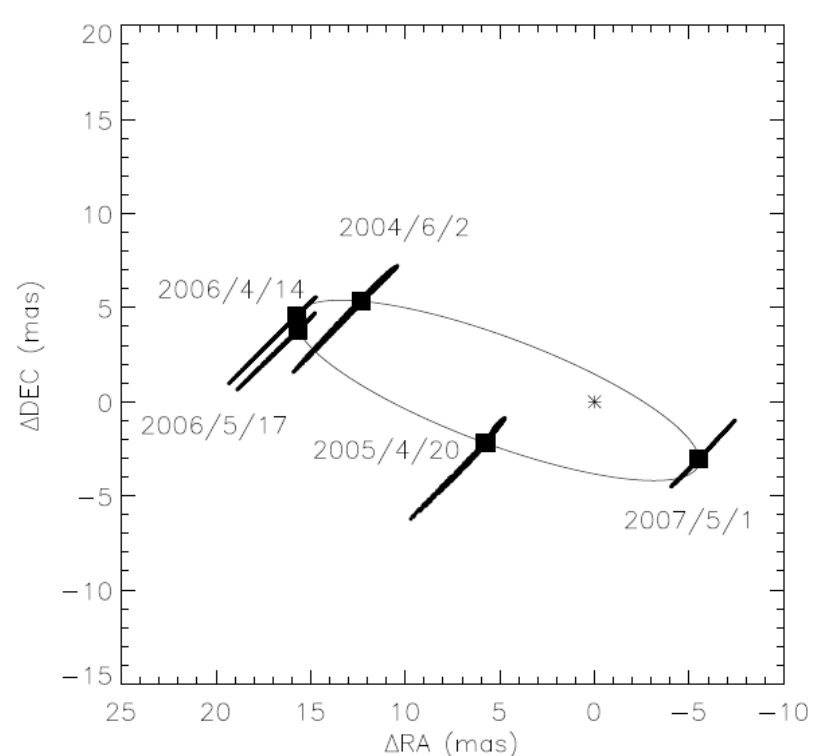

Figure 6 Haro 1-14c orbit (Schaefer et al., 2008)

\subsection{Circumstellar environment of Evolved Stars}

KI observations using grism mode were used to measure the stellar and molecular radii of the mira star R Vir from KI in grism mode (Eisner et al. 2007). The size of the emitting region varies dramatically in the 2.0-2.4 micron observing window. Simple models reproduce these wavelength-dependent variations using extended molecular layers, which absorb stellar radiation and re-emit it at longer wavelengths. The observations constrain the stellar photospheric radius, uncontaminated by molecular emission, and imply that most of the molecular opacity arises at approximately twice the radius of the stellar photosphere.
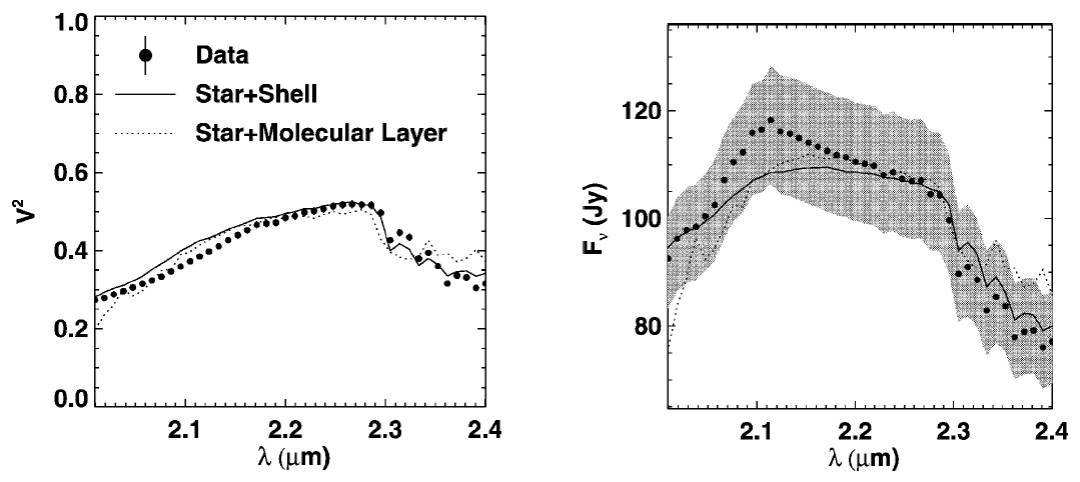

Figure 5 Observed and fluxes V2 plotted with the best-fit models (Eisner et al. 2007) 
Molecules such as CO, $\mathrm{H} 2 \mathrm{O}$ layers are evident from these observations. $\mathrm{H}_{2} \mathrm{O}$ opacity Dominates from $\sim 2.0-2.2 \mathrm{~mm}$ and 2.3-2.4 mm. Additional opacity from $\mathrm{CO}$ is evident in the wavelength region 2.3-2.4 mm. Photospheric emission dominated the region 2.2-2.3 mm. Models including a molecular shell plus a stellar photosphere can reproduce the data. These observations resolved emission from the stellar photosphere and molecular layers $\left(\mathrm{CO} \& \mathrm{H}_{2} \mathrm{O}\right)$ at approximately twice the stellar radius.

\section{FUTURE PLANS}

- ASTRA project: Starting July 2006, WMKO was awarded a \$2M Major Research Instrumentation grant from the National Science Foundation to upgrade the existing interferometer through the ASTtrometry and phaseReferencing Astronomy (ASTRA) project ${ }^{18}$. The goals of this project is to develop three new modes: a Self Phase Referencing mode (on-axis phase referencing for high spectral resolution observations), a Dual Field Phase Referencing mode (off-axis phase referencing for fainter observations), and an Astrometric mode. The Self Phase Referencing as been demonstrated on the sky recently and should be available to the community for shared-risk observations starting 2009A (see 09A call for proposal on the Michelson Science Center website).

- OHANA project: The 'OHANA project achieved first fringes on June 17, 2005. Since then, the instrumental setup at WMKO has been further improved: limiting magnitude has been pushed to $\mathrm{K} \sim 11.5$, and polarization control is better understood ${ }^{20}$. Efforts are underway to combine CFHT-GEMINI telescopes baselines ${ }^{21}$.

- Laser guide star (LGS) implementation on Keck 1: The Keck1 LGS developments is not part of KI project, but this capability would open-up the possibility of operating KI in laser guide star mode and hence observing highly obscured astronomical objects which are typically faint in the visible and bright in the infrared. Currently this operation is not possible since only Keck 2 telescope has LGS capability. The LGS capability for the Keck1 telescope is expected to be complete by the end of 2008. The near-future K1 LGS and KI astrometry capabilities would enable addressing interesting astrophysical problems through galactic center observations ${ }^{19}$.

\section{ACKNOWLEDGEMENTS}

The Keck Interferometer is funded by the National Aeronautics and Space Administration (NASA). Observations presented were obtained at the W. M. Keck Observatory, which is operated as a scientific partnership among the California Institute of Technology, the University of California, and NASA. The Observatory was made possible by the generous financial support of the W. M. Keck Foundation. Part of this work was performed at the Jet Propulsion Laboratory, California Institute of Technology, and at the Michelson Science Center, California Institute of Technology, under contract with NASA.

\section{REFERENCES}

[1] P. L. Wizinowich et al., 2006, "Recent progress at the Keck Interferometer," Proc. SPIE, 6268, 62680N.

[2] M. M. Colavita, P. L. Wizinowich, \& R. L. Akeson, 2006, "Nulling at the Keck Interferometer," Proc. SPIE, 6268, 626803.

[3] R. Millan-Gabet, 2006, “Keck Interferometer V2 science,” Proc. SPIE, 6268, 626800.

[4] P. L. Wizinowich, R. L. Akeson, et al., 2004, "Visibility science operations with the Keck Interferometer," Proc. SPIE, 5491, 1678.

[5] M. M. Colavita, P. L. Wizinowich, \& R. L. Akeson, 2004, "Keck Interferometer status and plans," Proc. SPIE, $5491,454$.

[6] R. L. Akeson, 2004, “Keck Interferometer science: present and future," Proc. SPIE, 5491, 28. 
[7] M. Colavita et al., 2008, "Keck Interferometer StatusRecent progress" Proc. SPIE, 7013, 7013-32 (this conference).

[8] R. K. Barry et al., 2008, "First science with the Keck interferometer nuller," Proc. SPIE, 7013, 7013-25 (this conference).

[9] Milliarcsecond N-Band Observations of the Nova RS Ophiuchi: First Science with the Keck Interferometer Nuller, R. Barry et al., 2008, Astrophysical Journal, 677, 1253

[10] E. Serabyn et al., 2006, "Science observations with the Keck Interferometer nuller," Proc. SPIE, 6268, 626815.

[11] J. A. Eisner, et al, 2007, "Stellar and Molecular Radii of a Mira Star: First Observations with the Keck Interferometer Grism",654,77

[12] J. A. Eisner, 2007, "Water vapour and hydrogen in the terrestrial-planet-forming region of a protoplanetary disk", Nature, 447, 562

[13] A. Tanner et al., 2007, "SIM PlanetQuest Key Project Precursor Observations to Detect Gas Giant Planets around Young Stars", PASP, 119, 747

[14] A. Boden et al, 2007, "Dynamical Masses for Pre-Main Sequence Stars: A Preliminary Physical Orbit for V773 Tau A", ApJ, 670, 1214

[15] J.A. Eisner et al, 2007, "Near-Infrared Interferometric, Spectroscopic, and Photometric Monitoring of T Tauri Inner Disks", ApJ, 669, 1072

[16] G. Schaefer et al, 2008, "Preliminary Orbit of the Young Binary Haro 1-14c", AJ, 2008, 135, 1659

[17] A. Boden et al, 2008, "Interferometric Evidence for Resolved Warm Dust in the DQ Tau System", ApJ, submitted

[18] J. M. Woillez, J.-U. Pott, P. L. Wizinowich, \& J. R. Graham, 2008, “ASTRA: The astrometric and phase-referencing astronomy upgrade for the Keck interferometer," Proc. SPIE, 7013, 7013-35 (this conference).

[19] E. Johansson et al., 2008, "Upgrading the Keck AO wavefront controllers", Proc. SPIE, 7015, 7013-121.

[20] J.-U. Pott et al., 2008, "The first IR fringes on Galactic Center sources: results in a new era of long baseline interferometry at the sensitivity limit of current larger aperture arrays," Proc. SPIE, 7013, 7013-73 (this conference).

[21] J. M. Woillez, 2008, "First demonstration of 'OHANA on the Keck-Keck baseline: results and implications" Proc. SPIE, 7013, 7013-32 (this conference).

[22] T. Kotani et al., 2008, "The FIRST project: a single-mode fiber-based, very high-dynamic range, diffraction-limited imaging instrument at visible to near-infrared wavelengths" Proc. SPIE, 7013, 7013-59 (this conference). 